\title{
¿A quién le importa el canto en el aula? Estudio basado en un cuestionario
}

Who cares about singing in the classroom? Study based on a questionnaire

\author{
Juan Rafael Muñoz Muñoz \\ irmunoz@ual.es \\ Departamento de Educación \\ Universidad de Almería \\ Almería, España \\ ORCID: http://orcid.org/0000-0002-6601-7329
}

doi: 10.7203/LEEME.44.1563

Recibido: 21-07-2019 Aceptado: 30-09-2019. Contacto y correspondencia: Juan Rafael Muñoz Muñoz, Departamento de Educación Universidad de Almería, C/ Argollones, $152^{\circ}$ C, C.P. 04004 Almería. España.

\section{Resumen}

Cantar es la actividad más importante en la Educación Musical en el ámbito educativo, sobre todo, en la Educación Infantil y Primaria. Algunos importantes autores han destacado la importancia del canto para la construcción del conocimiento musical y el desarrollo de la formación integral del infante en las primeras etapas educativas. Con este estudio se pretende conocer si al profesorado le gusta cantar en el aula y con qué periodicidad lo hace. También, se quiere constatar la importancia que le otorga el profesorado a esta actividad, así como la importancia que cree que le conceden sus compañeros, las familias, las editoriales, la administración educativa y la sociedad en general. En la metodología del estudio, se ha utilizado un cuestionario que han cumplimentado maestros de centros escolares de Almería, Granada y Sevilla. Se podrá observar que a los maestros les gusta cantar y lo hacen habitualmente en su aula. De igual modo, se pondrá de manifiesto que las familias y las editoriales conceden poca importancia al hecho de cantar en clase y que esta importancia es aún menor para la administración educativa y la sociedad en general.

Palabras clave: Educación Musical, cantar, Infantil, Primaria

\section{Abstract}

Singing is the most important activity in music education, particularly in the nursery and primary stages. Various authors have emphasized the importance of song in musical education and the overall education of children in the early stages of learning. This study aims to establish whether teachers enjoy singing in class and how often they do so. It also aims to determine how important they believe singing is and how important they believe it is to their colleagues, families, publishers, educational administrators, and society in general. The methodology used is a questionnaire completed by teachers in education centers in Almeria, Granada, and Seville. We can observe that teachers enjoy singing and often use song in the classroom. Conversely, it becomes clear that families and publishers attach little relevance to singing in the classroom and educational administrators and society in general have even less interest in these activities.

Key words: Musical Education, Singing, Elementary, Primary.

@JUAN RAFAEL MUÑOZ MUÑOZ. THE CONTENT OF THIS ARTICLE IS THE SOLE RESPONSIBILITY OF THE AUTHORS. THE REVISTA ELECTRÓNICA DE LEEME AND UNIVERSITAT DE VALĖNCIA ARE NOT LIABLE FOR ANY LEGAL ACTIONS THAT MAY ARISE INVOLVING THE ARTICLE'S CONTENT. REVISTA ELECTRÓNICA DE LEEME - LISTA ELECTRÓNICA EUROPEA DE MÚSICA EN LA EDUCACIÓN-HTTP://OJS.UV.ES/INDEX/PHP/LEEME/INDEX. ISSN: 1575-9563. EDITORES: UNIVERSIDAD DE VALENCIA Y JESÚS TEJADA GIMÉNEZ. VISIBILIDAD DE ESTA REVISTA: SCOPUS, EMERGING SOURCES CITATION INDEX (CLARIVATE), EBSCO, CINDOC (CSIC), CITEFACTOR, COPAC, DIALNET, DICE (CSIC), DOAJ, E-REVISTAS (CSIC), EBSCO PREMIER, ERIH+, GALE CENGAGE LEARNING, IN-RECS, IRESIE, LATINDEX, MIAR, OCLC WORLDCAT, RESH, REDIB, RILM CORE JOURNALS, SUDOC, ULRICHS, ESTA REVISTA ESTA PUBLICADA CON EL APOYO INSTITUCIONAL DE REDIRIS-CONSEJO SUPERIOR DE INVESTIGACIONES CIENTIFICAS Y ES DE ACCESO LIBRE. CREATIVE COMMONS LICENSE 4.O BY 


\section{Introducción}

El canto es la principal actividad musical de cuantas se desarrollan en la Educación Infantil y Primaria. Las canciones nos han acompañado en las aulas desde siempre. Tradicionalmente, gran parte de las canciones que se cantaban habían sido aprendidas a través de nuestros abuelos, y de los juegos que se realizaban en las calles y plazas de los pueblos y las ciudades (Cerrillo, 2005). Sin embargo, su práctica (Cámara, 2004) se ha reducido sustancialmente haciendo que el alumnado cante menos. Una de las razones se encuentra en el aumento de abuelas y abuelos que ya no viven en las casas de sus hijos y sus nietos y, por lo tanto, ya no enseñan las canciones de siempre (Cerrillo, 2010). Otros contextos familiares, considerados cauces naturales del aprendizaje de las canciones como celebraciones, reuniones fiestas, etc. también se han ido perdiendo (Díaz, 2007) Por otra parte, también hay que destacar (Zamora, 2002) como la televisión, las vídeo-consolas y los ordenadores han centrado la atención del infante haciendo que se olvide de los juegos populares y de sus canciones. En realidad, lo que se ha producido es un cambio en la utilización y finalidad del canto en el aula que, a la vez, ha determinado un cambio en los repertorios. Así, se observa una mayor presencia de canciones de autor, que se han ido popularizando con el tiempo y, junto a ellas, canciones seleccionadas en función de objetivos didácticos (Hernández, 2012). De esta forma, las aulas disponen de un repertorio más amplio que hacen que convivan músicas de diferentes géneros, épocas y estilos (Bernal y Calvo, 2004).

Para el fomento de la práctica del canto es fundamental la disposición hacia esta actividad de los maestros, así como del educando. Eso hace que se puedan plantear algunas preguntas: ¿le gusta cantar a los maestros? ¿Les gusta cantar a sus estudiantes? ¿Se canta en las aulas? ¿Qué importancia se le da al hecho cantar en el aula? Las respuestas pueden encontrarse en el propio profesorado. El presente trabajo quiere abordar estas cuestiones desde la perspectiva y la opinión de un amplio grupo de ellos. En este estudio, se pretende investigar si al profesorado le gusta cantar y si a su alumnado también le gusta cantar. Paralelamente, queremos conocer si la actividad de canto la desarrollan diariamente, habitualmente o simplemente a veces. Además, es de interés en este estudio conocer cuál es la importancia que según los maestros se le da al hecho de cantar, por parte de los propios maestros, sus compañeros, las familias y, por otra parte, dentro del ámbito institucional, la importancia que le otorgan las editoriales, la administración educativa y la sociedad en general. Todo ello se enmarca dentro de un estudio más amplio centrado en la presencia de la canción popular infantil en la escuela y los repertorios que en la actualidad se utilizan en las aulas de Educación Infantil y Educación Primaria.

@JUAN RAFAEL MUÑOZ MUÑOZ. THE CONTENT OF THIS ARTICLE IS THE SOLE RESPONSIBILITY OF THE AUTHORS. THE REVISTA ELECTRÓNICA DE LEEME AND UNIVERSITAT DE VALĖNCIA ARE NOT LIABLE FOR ANY LEGAL ACTIONS THAT MAY ARISE INVOLVING THE ARTICLE'S CONTENT. REVISTA ELECTRÓNICA DE LEEME -LISTA ELECTRÓNICA EUROPEA DE MÚSICA EN LA EDUCACIÓN.HTTP:// /OJS.UV.ES/INDEX/PHP/LEEME/INDEX. ISSN: 1575-9563. EDITORES: UNIVERSIDAD DE VALENCIA Y JESÚS TEJADA GIMÉNEZ. VISIBILIDAD DE ESTA REVISTA: SCOPUS, EMERGING SOURCES CITATION INDEX (CLARIVATE), EBSCO, CINDOC (CSIC), CITEFACTOR, COPAC, DIALNET, DICE (CSIC), DOAJ, E-REVISTAS (CSIC), EBSCO PUBLICADA CON EL APOYO INSTITUCIONAL DE REDIRIS-CONSEJO SUPERIOR DE INVESTIGACIONES CIENTIFICAS Y ES DE ACCESO LIBRE. CREATIVE COMMONS LICENSE 4.0 BY 


\subsection{La importancia del canto en el aula}

La presencia del canto en la vida del infante tiene lugar mucho antes de que llegue a la escuela. Su trascendencia adquiere una especial relevancia desde los primeros meses de vida, en el ámbito familiar, cuando actúa como un singular medio de relación y comunicación con sus familiares próximos (Morant, 1999). Paralelamente, el canto constituye la principal base de una sólida cultura musical por encima de otras manifestaciones musicales utilizando la voz como el instrumento accesible a todos, como han señalado diferentes pedagogos musicales entre los que podemos destacar a Kodaly (Subirats, 2007). A través de los tiempos, el canto ha permitido exteriorizar los sentimientos, las sensaciones, los miedos y los diferentes estados de ánimo. Hasta tal punto que: "Se puede decir, sin temor a equivocarse, que el canto es el principio y el alma de la música" (Willems, 1981, p.140).

En el ámbito educativo, Muñoz Muñoz (2017) considera la canción como el contexto de trabajo musical más completo y globalizador de cuantos podemos disponer en el aula, capaz de integrar el tratamiento de capacidades y contenidos musicales y extramusicales. Se utiliza como medio para el estudio de los elementos de la música (Bernal y Calvo, 2004), el desarrollo de la audiopercepción (Monks, 2003) y de la psicomotricidad (Phillips, 1992), favoreciendo la formación integral del infante (Cámara, 2005; Díaz, 2011; Giraldez, 2014; Sigcha, Constante, Defaz, Trávez, y Ceíro, 2016), así como su uso para potenciar el desarrollo de otras capacidades como la memorización y distintas habilidades cognitivas (Selfa, 2019). Dentro de estas últimas, para abordar contenido de las diferentes áreas de conocimiento (López de la Calle, 2007; Martín Escobar, 2010; Casals, Carrillo y González-Martín, 2014). Existen otras posibilidades de utilización de las canciones como medio para la animación a la lectura desde la educación infantil (Muñoz Muñoz, 2017) y como activadoras de la educación emocional del infante (Bisquerra, 2011).

La Música favorece la educación en valores en los distintos niveles educativos (Tiburcio, 2010; Conejo, 2012) y la inclusión (Fautley y Daubney, 2018). Por medio de las canciones y el canto se potencia la educación intercultural y el respeto a los demás sin diferencias de razas, sexo o religión (Bernabé, 2013), contribuyendo a la formación del infante en educación para la paz y la no violencia potenciando actitudes y comportamientos propios de una cultura de paz (Cabedo y Arriaga, 2016). Paralelamente, la realización de actividades musicales grupales, incluidas las de canto, ofrece la vivencia de aspectos que tiene que ver con la vida social, emocional y física de quiénes intervienen (Cunha y Lorenzino, 2012). Todo ello se puede plantear desde la Educación Infantil y, posteriormente, ampliarse a lo largo de la Educación Primaria (Moya, Hernández Bravo, Hernández, y Cózar, 2014). 


\section{Método}

La intención de este estudio, y por tanto su objetivo principal, es conocer con que énfasis se presenta el canto en las aulas de los colegios andaluces, según la opinión de los maestros, y la importancia que se concede a esta actividad. Como objetivos específicos se plantean los siguientes:

- Constatar cuál es la disposición de los maestros y del alumnado respecto al hecho de cantar en el aula.

- Reconocer con que periodicidad el canto se lleva a cabo en las aulas de los centros de infantil y primaria.

- Comprobar la importancia que conceden al hecho de cantar en el aula el profesorado, sus compañeros, las familias, las editoriales, la administración educativa y la sociedad en general.

\subsection{Muestra}

La población estaría integrada por el conjunto de docentes de Educación Infantil y Primaria que impartía docencia en los colegios públicos y privados de las provincias de Almería, Granada y Sevilla. Inicialmente, se determinó que esta investigación estaría centrada en la provincia de Almería; después, se pensó que sería interesante incluir a profesorado de otras provincias andaluzas para comprobar si los resultados eran similares en cada una de ellas. Se decidió pasar los cuestionarios en otras dos provincias andaluzas, elegidas al azar, puesto que pretendíamos obtener una información más completa en la Comunidad Autónoma Andaluza. El resultado determinó que las provincias fueran: Almería, Granada y Sevilla; y, como quiera que las poblaciones de esta investigación son muy numerosas y resultaría bastante complicado estudiar a todos sus integrantes se ha hecho necesario elaborar una muestra. Se ha utilizado un muestreo no probabilístico, concretamente un muestreo voluntario (McMillan y Schumacher 2010, p.143) puesto que los docentes participaron de manera voluntaria en la investigación.

Con el objeto de extraer la información más representativa del conjunto del profesorado de Educación Infantil y Primaria de las provincias de Almería, Granada y Sevilla, se ha optado por incluir en la muestra teórica a todos los centros públicos y concertados. Consultados los datos estadísticos publicados por el Ministerio de Educación, Cultura y Deporte (2016) y la Consejería de Educación de la Junta de Andalucía (2016) correspondientes a estas provincias, y siguiendo las indicaciones de García Ferrando (1985, p.142), atendiendo a su fórmula de cálculo y la población que debíamos considerar, la muestra tendría que situarse en 302 docentes. La muestra real que se ha obtenido ha sido de 307 docentes. Esta muestra presenta un margen de error del 5,6\%, atendiendo al tamaño de la muestra y un nivel de confianza del $95 \%$.

Tal como precisa Fox (1981, pp.367-369) a propósito del tamaño de la muestra, se podría decir que: 
a) Muestra invitada. Se ha invitado a un total de 800 docentes.

b) Muestra aceptante. Han aceptado contestar la encuesta un total de 307 docentes.

c) Muestra productora de datos. La constituyen los 307 maestros que han contestado.

Observando los docentes que han respondido a los cuestionarios, se puede constatar que en Almería lo han hecho 182 docentes, y otros 125 docentes entre el profesorado de Granada y Sevilla, garantizando que el número en cada una de estas provincias fuera superior a 50 encuestas: 58 encuestas en la provincia de Granada y 67 en la provincia de Sevilla. En cuanto a los centros a los que pertenece el profesorado que ha respondido a los cuestionarios, se encuentran: Centros Públicos de Infantil, Centros Públicos de Infantil y Primaria, Colegios Públicos Rurales y Centros Concertados de Educación Infantil y Educación Primaria. Hay que recordar que la distribución de las encuestas se ha hecho de forma aleatoria entre el profesorado, de las tres provincias mencionadas, sin tener en cuenta el centro en el que desarrolla su labor docente.

Tabla 1. Tipología de los centros de procedencia de los encuestados

\begin{tabular}{llllll}
\hline & $\begin{array}{l}\text { Público Infantil } \\
\text { y Primaria }\end{array}$ & Público Infantil & CPR & Concertado & TOTAL \\
\hline ALMERÍA & 134 & 14 & 18 & 16 & 182 \\
GRANADA & 41 & 3 & 6 & 8 & 58 \\
SEVILLA & 54 & 13 & 0 & 0 & 67 \\
TOTAL & 229 & 30 & 24 & 24 & 307 \\
\hline
\end{tabular}

Fuente: elaboración propia

Cada cuestionario iba en un sobre cerrado para asegurar el anonimato de los participantes y acompañadas de una carta explicativa en la que se pedía su participación y se explicaba la finalidad de la investigación. La mayoría de informantes han sido mujeres, ya que apenas se han recogido el $16 \%$ de los cuestionarios de hombres.

Tabla 2. Sexo de los encuestados

\begin{tabular}{lll}
\hline & Frecuencia & Porcentaje \\
\hline Hombres & 49 & $16 \%$ \\
Mujeres & 258 & $84 \%$ \\
Total & 307 & $100 \%$ \\
\hline
\end{tabular}

Fuente: elaboración propia

En cuanto a la edad de los encuestados, se puede comprobar que el mayor número de del profesorado que ha respondido, que suponen un $32.2 \%$, tienen una edad comprendida entre los 31-40 años. Le sigue en número, aquellos docentes cuya edad se sitúa entre los 20-30 años

@JUAN RAFAEL MUÑOZ MUÑOZ. THE CONTENT OF THIS ARTICLE IS THE SOLE RESPONSIBILITY OF THE AUTHORS. THE REVISTA ELECTRÓNICA DE LEEME AND UNIVERSITAT DE VALĖNCIA ARE NOT LIABLE FOR ANY LEGAL ACTIONS THAT MAY ARISE INVOLVING THE ARTICLE'S CONTENT. REVISTA ELECTRÓNICA DE LEEME -LISTA ELECTRÓNICA EUROPEA DE MÚSICA EN LA EDUCACIÓN.HTTP://OJS.UV.ES/INDEX/PHP/LEEME/INDEX. ISSN: 1575-9563. EDITORES: UNIVERSIDAD DE VALENCIA Y JESÚS TEJADA GIMÉNEZ. VISIBILIDAD DE ESTA REVISTA: SCOPUS, EMERGING SOURCES CITATION INDEX (CLARIVATE), EBSCO, CINDOC (CSIC), CITEFACTOR, COPAC, DIALNET, DICE (CSIC), DOAJ, E-REVISTAS (CSIC), EBSCO PUBLICADA CON EL APOYO INSTITUCIONAL DE REDIRIS-CONSEJO SUPERIOR DE INVESTIGACIONES CIENTIFICAS Y ES DE ACCESO LIBRE. CREATIVE COMMONS LICENSE 4.O BY 
con un $29.0 \%$. El menor número de encuestados por edad se encuentra en la franja comprendida entre los 51 y más.

Tabla 3. Edad de los encuestados

\begin{tabular}{lcc}
\hline & Frecuencia & Porcentaje \\
\hline $20-30$ & 90 & $29.0 \%$ \\
$31-40$ & 99 & $32.2 \%$ \\
$41-50$ & 73 & $24.1 \%$ \\
51 y más & 29 & $9.5 \%$ \\
En blanco & 16 & $5.2 \%$ \\
Total & 307 & $100 \%$ \\
\hline
\end{tabular}

Fuente: elaboración propia

\subsection{Instrumento}

Para la elaboración de las preguntas del cuestionario, se han tenido en cuenta otras investigaciones realizadas en otros estudios en el ámbito de esta investigación (Oriol, 2002; Chao, 2005), trabajos que además han permitido establecer la validez de elaboración de las preguntas formuladas. En el trabajo de Oriol (2002), se analizan las propuestas didácticas del folclore que realiza el profesorado de los colegios de Educación Primaria de la Comunidad de Madrid, a través de un cuestionario que cumplimentaron 145 maestros especialistas en música. Por su parte, Chao (2005) pretende conocer si el profesorado que imparte música en los colegios de educación primaria en Galicia utiliza habitualmente en su práctica docente el folclore musical gallego. También, se han considerado las aportaciones de Estévez (2008), quien realiza un análisis sobre la música popular gallega de cara a su utilización didáctica, estableciendo las conexiones adecuadas con las diferentes materias que constituyen el currículum escolar. Del mismo modo, se han contemplado las aportaciones de los cuestionarios utilizados en la investigación de Martín Escobar (2010), que se centra en la presencia de las canciones de tradición oral en la Región de Murcia.

Para asegurar la validez de la evidencia del cuestionario, se ha aplicado la técnica denominada "juicio de expertos". Han participado un total de siete expertos: uno de la Universidad de León, otro de la Universidad Autónoma de Madrid, un tercer experto de la Universidad del País Vasco, el cuarto de la Universidad de Granada y tres más de la Universidad de Almería. Se considera que ha sido buena la confiabilidad de la escala con un coeficiente alfa de Cronbach $\alpha=0.795$. Se tuvieron en cuenta las propuestas de los expertos. Después, el cuestionario pasó las correspondientes pruebas piloto llegando a gozar de la satisfacción del profesorado que participó como voluntario.

Las características de las preguntas incluidas en el cuestionario completo corresponden a diferentes tipos según la información que se ha solicitado al profesorado. De esta forma, siguiendo la propuesta de Blaxter, Hughes y Tight (2000, p. 218), se han encontrado preguntas

@JUAN RAFAEL MUÑOZ MUÑOZ. THE CONTENT OF THIS ARTICLE IS THE SOLE RESPONSIBILITY OF THE AUTHORS. THE REVISTA ELECTRÓNICA DE LEEME AND UNIVERSITAT DE VALĖNCIA ARE NOT LIABLE FOR ANY LEGAL ACTIONS THAT MAY ARISE INVOLVING THE ARTICLE'S CONTENT. REVISTA ELECTRÓNICA DE LEEME - LISTA ELECTRÓNICA EUROPEA DE MÚSICA EN LA EDUCACIÓN-HTTP://OJS.UV.ES/INDEX/PHP/LEEME/INDEX. ISSN: 1575-9563. EDITORES: UNIVERSIDAD DE VALENCIA Y JESÚS TEJADA GIMÉNEZ. VISIBILIDAD DE ESTA REVISTA: SCOPUS, EMERGING SOURCES CITATION INDEX (CLARIVATE), EBSCO, CINDOC (CSIC), CITEFACTOR, COPAC, DIALNET, DICE (CSIC), DOAJ, E-REVISTAS (CSIC), EBSCO PREMIER, ERIH+, GALE CENGAGE LEARNING, IN-RECS, IRESIE, LATINDEX, MIAR, OCLC WORLDCAT, RESH, REDIB, RILM CORE JOURNALS, SUDOC, ULRICHS, ESTA REVISTA ESTA PUBLICADA CON EL APOYO INSTITUCIONAL DE REDIRIS-CONSEJO SUPERIOR DE INVESTIGACIONES CIENTIFICAS Y ES DE ACCESO LIBRE. CREATIVE COMMONS LICENSE 4.O BY 
de: "cantidad o información puntual, categoría, lista o elección múltiple, escala, cuadrícula o tabla completa y final abierto". Los cuestionarios a los que ha respondido el profesorado han sido tratados con el Programa de IBM SPSS, versión Windows.

\subsection{Procedimiento}

El procedimiento que se ha utilizado ha sido la visita personal a los colegios y Centros de Profesorado para contactar con los docentes que se prestaron primero a distribuir y, posteriormente, a recoger las encuestas de los docentes de su centro. En cuanto a la metodología utilizada para poder desarrollar los objetivos planteados, se ha utilizado un estudio descriptivo con datos cuantitativos. Se ha llevado a cabo el estudio a través de una encuesta de corte cuantitativo, para comprobar el grado de relación de las diferentes variables que se pueden considerar en ellos como: el sexo, la edad, la titulación o el nivel educativo.

\section{Resultados}

El análisis de las respuestas obtenidas en la primera pregunta ha revelado que a la mayoría del profesorado le gusta cantar en el aula. Del mismo modo, se puede observar cómo el porcentaje de profesorado que ha manifestado que no les gusta cantar en clase no llega al $8 \%$ (Tabla 4):

Tabla 4. Resultados pregunta 1 “Le gusta cantar en clase?”

\begin{tabular}{ccc}
\hline & Frecuencia & Porcentaje \\
\hline Sí & 281 & $91.5 \%$ \\
No & 24 & $7.8 \%$ \\
Ns/Nc & 2 & $0.7 \%$ \\
Total & 307 & $100 \%$ \\
\hline
\end{tabular}

Fuente: elaboración propia

En todas las provincias en las que se ha realizado el cuestionario, el profesorado afirma que le gusta cantar en clase (Sevilla: 95.5\%; Almería: 90.7\%, y Granada: 89.7\%), como puede observarse en la Tabla 5:

Tabla 5. Relación Provincias - pregunta 1 “Le gusta cantar en clase?”

\begin{tabular}{cccc}
\hline & Almería & Granada & Sevilla \\
\hline \multirow{2}{*}{ Sí } & 165 & 52 & 64 \\
& $90.7 \%$ & $89.7 \%$ & $95.5 \%$ \\
No & 15 & 6 & 3 \\
Ns/Nc & $8.2 \%$ & $10.3 \%$ & $4.5 \%$ \\
& 2 & 0 & 0
\end{tabular}




\begin{tabular}{cccc}
\hline & $1.1 \%$ & $0.0 \%$ & $0.0 \%$ \\
Totales & 182 & 58 & 67 \\
& $100 \%$ & $100 \%$ & $100 \%$ \\
\hline
\end{tabular}

Fuente: elaboración propia

Atendiendo al nivel educativo (Tabla 6), los resultados han revelado que a medida que aumenta el nivel educativo es menor el número de maestros que cantan (significatividad asintótica $\chi^{2}=0.001$ ). Esta realidad se confirma al cruzar la variable titulación con esta pregunta (significatividad asintótica $\chi^{2}=0.000$ ), puesto que a las maestras que más les gusta cantar son los de Educación Infantil.

Tabla 6. Relación Nivel Educativo - pregunta 1 “Le gusta cantar en clase?”

\begin{tabular}{ccccccc}
\hline & Infantil & $1^{\circ} \mathrm{y} 2^{\circ}$ & $3^{\circ} \mathrm{y} 4^{\circ}$ & $5^{\circ}$ y $6^{\circ}$ & Todos & Total \\
\hline \multirow{2}{*}{ Sí } & 134 & 37 & 23 & 10 & 28 & 232 \\
& $57.8 \%$ & $15.9 \%$ & $9.9 \%$ & $4.3 \%$ & $12.1 \%$ & $100 \%$ \\
No & 2 & 7 & 4 & 4 & 1 & 18 \\
& $11.2 \%$ & $38.9 \%$ & $22.3 \%$ & $22.3 \%$ & $5.6 \%$ & $100 \%$ \\
Total & 136 & 44 & 27 & 14 & 29 & 250 \\
& $54.4 \%$ & $17.6 \%$ & $10.8 \%$ & $5.6 \%$ & $11.6 \%$ & $100 \%$ \\
\hline
\end{tabular}

Fuente: elaboración propia

Esta realidad se confirma al cruzar la variable titulación con esta pregunta (significatividad asintótica $\chi^{2}=0.000$ ), puesto que el profesorado que más gusta del canto es el de Educación Infantil (Tabla 7).

Tabla 7. Relación Titulación - pregunta 1 “Le gusta cantar en clase?”

\begin{tabular}{cccccc}
\hline & Ed. Infantil & Ed. Primaria & Ed. Musical & Otras & Total \\
\hline \multirow{2}{*}{ Sí } & 132 & 30 & 44 & 51 & 257 \\
& $51.4 \%$ & $11.7 \%$ & $17.1 \%$ & 19.8 & $100 \%$ \\
No & 4 & 5 & 1 & 11 & 21 \\
& $19.0 \%$ & $23.8 \%$ & $4.8 \%$ & 52.4 & $100 \%$ \\
Totales & 136 & 35 & 45 & 62 & 278 \\
& $48.9 \%$ & $12.6 \%$ & $16.2 \%$ & 22.3 & $100 \%$ \\
\hline
\end{tabular}

Fuente: elaboración propia

Por otra parte, se ha querido comprobar si la actividad de canto, al ser una actividad que gusta al profesorado, se encuentra por ello presente en las aulas. Por lo tanto, se ha preguntado: ¿Canta usted en clase? El análisis de los datos obtenidos a esta pregunta ha confirmado que prácticamente el $80 \%$ de los maestros entrevistados han señalado que cantan en clase diariamente o habitualmente. Por otra parte, el profesorado que no canta nunca en clase está por debajo del $4 \%$.

@JUAN RAFAEL MUÑOZ MUÑOZ. THE CONTENT OF THIS ARTICLE IS THE SOLE RESPONSIBILITY OF THE AUTHORS. THE REVISTA ELECTRÓNICA DE LEEME AND UNIVERSITAT DE VALËNCIA ARE NOT LABLE FOR ANY LEGA ACTIONS THAT MAY ARISE INVOLVING THE ARTICLE'S CONTENT. REVISTA ELECTRÓNCA DE LEEME - LISTA ELECTRÓNICA EUROPEA DE MÚSICA EN LA EDUCACIÓN-HTTP://OJS.UV.ES/INDEX/PHP/LEEME/INDEX. ISSN: 1575-9563. EDITORES: UNIVERSIDAD DE VALENCIA Y JESÚS TEJADA GIMÉNEZ. VISIBILIDAD DE ESTA REVISTA: SCOPUS, EMERGING SOURCES CITATION INDEX (CLARIVATE), EBSCO, CINDOC (CSIC), CITEFACTOR, COPAC, DIALNET, DICE (CSIC), DOAJ, E-REVISTAS (CSIC), EBSCO PREMIER, ERIH+, GALE CENGAGE LEARNING, IN-RECS, IRESIE, LATINDEX, MIAR, OCLC WORLDCAT, RESH, REDIB, RILM CORE JOURNALS, SUDOC, ULRICHS, ESTA REVISTA ESTÁ PUBLICADA CON EL APOYO INSTITUCIONAL DE REDIRIS-CONSEJO SUPERIOR DE INVESTIGACIONES CIENTIFICAS Y ES DE ACCESO LIBRE. CREATIVE COMMONS LICENSE 4.O BY 
Tabla 8. Resultados pregunta 2 “Canta usted en clase?”

\begin{tabular}{ccc}
\hline & Frecuencia & Porcentaje \\
\hline Diariamente & 166 & $54.1 \%$ \\
Habitualmente & 77 & $25.1 \%$ \\
A veces & 51 & $16.6 \%$ \\
Nunca & 12 & $3.9 \%$ \\
NS/NC & 1 & $0.3 \%$ \\
\hline
\end{tabular}

Fuente: elaboración propia

Por provincias, los resultados muestran cómo al menos el $74 \%$ del profesorado de todas las provincias canta en clase diariamente o habitualmente. Paralelamente, se observa como las maestras y los maestros que no lo hacen no llegan al 4.5\% (Tabla 9).

Tabla 9. Relación Provincias - pregunta 2 “Canta usted en clase?”

\begin{tabular}{cccc}
\hline & Almería & Granada & Sevilla \\
\hline Diariamente & 95 & 34 & 37 \\
& $52.2 \%$ & $58.6 \%$ & $55.2 \%$ \\
Habitualmente & 48 & 9 & 20 \\
& $26.4 \%$ & $15.5 \%$ & $29.9 \%$ \\
A veces & 31 & 12 & 8 \\
& $17.0 \%$ & $20.7 \%$ & $11.9 \%$ \\
Nunca & 8 & 2 & 2 \\
& $4.4 \%$ & $3.4 \%$ & $3.0 \%$ \\
NS/NC & 0 & 1 & 0 \\
& $0.0 \%$ & $1.7 \%$ & $0.0 \%$ \\
Totales & 182 & 58 & 67 \\
& $100 \%$ & $100 \%$ & $100 \%$ \\
\hline
\end{tabular}

Fuente: elaboración propia

Considerando la edad de los encuestados (Tabla 10) se puede observar que, a medida que va aumentando la edad del profesorado, disminuye el número de maestros que cantan diariamente o habitualmente en clase. Los resultados estadísticos vienen a confirmar la significatividad de las respuestas (significatividad asintótica $\chi^{2}=0.041$ ).

Tabla 10. Relación Edad- pregunta 2 “CCanta usted en clase?”

\begin{tabular}{cccccc}
\hline & $20-30$ & $31-40$ & $41-50$ & $51-60$ & Total \\
\hline \multirow{2}{*}{ Diariamente } & 52 & 55 & 36 & 14 & 157 \\
& $33.2 \%$ & $35.0 \%$ & $22.9 \%$ & $8.9 \%$ & $100 \%$ \\
Habitualmente & 28 & 21 & 21 & 6 & 76 \\
& $36.9 \%$ & $27.7 \%$ & $27.7 \%$ & $7.9 \%$ & $100 \%$ \\
A veces & 11 & 22 & 10 & 7 & 50 \\
& $22.0 \%$ & $44.0 \%$ & $20.0 \%$ & $14.0 \%$ & $100 \%$ \\
Nunca & 2 & 1 & 7 & 2 & 12 \\
& $16.6 \%$ & $8.3 \%$ & $58.3 \%$ & $16.7 \%$ & $100 \%$ \\
Total & 93 & 99 & 74 & 29 & 295 \\
& $29.5 \%$ & $33.5 \%$ & $25.0 \%$ & $9.8 \%$ & $100 \%$ \\
\hline
\end{tabular}

Fuente: elaboración propia

@JUAN RAFAEL MUÑOZ MUÑOZ. THE CONTENT OF THIS ARTICLE IS THE SOLE RESPONSIBILITY OF THE AUTHORS. THE REVISTA ELECTRÓNICA DE LEEME AND UNIVERSITAT DE VALĖNCIA ARE NOT LIABLE FOR ANY LEGAL ACTIONS THAT MAY ARISE INVOLVING THE ARTICLE'S CONTENT. REVISTA ELECTRÓNICA DE LEEME -LISTA ELECTRÓNICA EUROPEA DE MÚSICA EN LA EDUCACIÓN.HTTP://OJS.UV.ES/INDEX/PHP/LEEME/INDEX. ISSN: 1575-9563. EDITORES: UNIVERSIDAD DE VALENCIA Y JESÚS TEJADA GIMÉNEZ. VISIBILIDAD DE ESTA REVISTA: SCOPUS, EMERGING SOURCES CITATION INDEX (CLARIVATE), EBSCO, CINDOC (CSIC), CITEFACTOR, COPAC, DIALNET, DICE (CSIC), DOAJ, E-REVISTAS (CSIC), EBSCO PREMIER, ERIH+, GALE CENGAGE LEARNING, IN-RECS, IRESIE, LATINDEX, MIAR, OCLC WORLDCAT, RESH, REDIB, RILM CORE JOURNALS, SUDOC, ULRICHS, ESTA REVISTA
PUBLICADA CON EL APOYO INSTITUCIONAL DE REDIRIS-CONSEJO SUPERIOR DE INVESTIGACIONES CIENTIFICAS Y ES DE ACCESO LIBRE. CREATIVE COMMONS LICENSE 4.0 BY 
La relación entre la variable nivel educativo en el que imparten sus clases los encuestados y la pregunta 2 “Canta usted en clase?” (Tabla 11), el mayor número de maestros que cantan corresponden a la Educación Infantil, seguidos de los maestros de $1^{\circ}$ y $2^{\circ}$ curso. Los que menos cantan en clase son los maestros que imparten sus clases en el $5^{\circ}$ y $6^{\circ}$ curso (significatividad asintótica $\chi^{2}=0.000$ ).

Tabla 11. Relación Nivel Educativo - pregunta 2 “Canta usted en clase?”

\begin{tabular}{ccccccc}
\hline & Ed. & $1^{\circ} \mathrm{y} 2^{\circ}$ & $3^{\circ} \mathrm{y} 4^{\circ}$ & $5^{\circ} \mathrm{y} 6^{\circ}$ & Todos & Total \\
\hline Infantil & 100 & 12 & 6 & 1 & 16 & 135 \\
Diariamente & $74.1 \%$ & $8.9 \%$ & $4.5 \%$ & $0.7 \%$ & $11.9 \%$ & $100 \%$ \\
& 30 & 15 & 8 & 2 & 8 & 63 \\
Habitualmente & $47.7 \%$ & $23.8 \%$ & $12.7 \%$ & $3.2 \%$ & $12.7 \%$ & $100 \%$ \\
& 6 & 14 & 12 & 5 & 5 & 42 \\
A veces & $14.3 \%$ & $33.4 \%$ & $28,6 \%$ & $11.9 \%$ & $11.9 \%$ & $100 \%$ \\
& 0 & 3 & 2 & 5 & 0 & 10 \\
Nunca & $0.0 \%$ & $30.0 \%$ & $20.0 \%$ & $50.0 \%$ & $0.0 \%$ & $100 \%$ \\
& 136 & 44 & 28 & 13 & 29 & 250 \\
Total & $54.4 \%$ & $17.6 \%$ & $11.2 \%$ & $5.2 \%$ & $11.6 \%$ & $100 \%$ \\
\hline
\end{tabular}

Fuente: elaboración propia

La variable titulación ha resultado significativa en el caso de las maestras de Educación Infantil, con un $61.2 \%$, son los que más cantan (significatividad asintótica $\chi^{2}=0.000$ ) (Tabla 12):

Tabla 12. Relación Titulación - pregunta 2 “Canta usted en clase?”

\begin{tabular}{cccccc}
\hline & Ed. & Ed. Primaria & Ed. Musical & Otras & Total \\
\hline Infantil & 93 & 10 & 26 & 23 & 152 \\
Diariamente & $61.2 \%$ & $6.6 \%$ & $17.1 \%$ & $15.1 \%$ & $100 \%$ \\
Habitualmente & 32 & 10 & 16 & 12 & 70 \\
A veces & $45.7 \%$ & $14.3 \%$ & $22.9 \%$ & $17.1 \%$ & $100 \%$ \\
& 11 & 12 & 2 & 21 & 46 \\
Nunca & $23.9 \%$ & $26.1 \%$ & $4.3 \%$ & $45.7 \%$ & $100 \%$ \\
& 1 & 3 & 0 & 7 & 11 \\
Totales & $9.1 \%$ & $27.3 \%$ & $0.0 \%$ & $63.6 \%$ & $100 \%$ \\
& 137 & 35 & 44 & 63 & 279 \\
& $49.1 \%$ & $12.5 \%$ & $15.8 \%$ & $22.6 \%$ & $100 \%$ \\
\hline
\end{tabular}

Fuente: elaboración propia

Por otra parte, se ha constatado que, según las maestras, a las niñas y a los niños les gusta cantar en clase mucho o bastante (Tabla 13). Resulta significativo como a juicio del profesorado no hay niñas o niños a los que no les guste cantar en clase.

@JUAN RAFAEL MUÑOZ MUÑOZ. THE CONTENT OF THIS ARTICLE IS THE SOLE RESPONSIBILITY OF THE AUTHORS. THE REVISTA ELECTRÓNICA DE LEEME AND UNIVERSITAT DE VALĖNCIA ARE NOT LIABLE FOR ANY LEGAL ACTIONS THAT MAY ARISE INVOLVING THE ARTICLE'S CONTENT. REVISTA ELECTRÓNICA DE LEEME -LISTA ELECTRÓNICA EUROPEA DE MÚSICA EN LA EDUCACIÓN-HTTP://OJS.UV.ES/INDEX/PHP/LEEME/INDEX. ISSN: 1575-9563. EDITORES: UNIVERSIDAD DE VALENCIA Y JESÚS TEJADA GIMÉNEZ. VISIBILIDAD DE ESTA REVISTA: SCOPUS, EMERGING SOURCES CITATION INDEX (CLARIVATE), EBSCO, CINDOC (CSIC), CITEFACTOR, COPAC, DIALNET, DICE (CSIC), DOAJ, E-REVISTAS (CSIC), EBSCO PREMIER, ERIH+, GALE CENGAGE LEARNING, IN-RECS, IRESIE, LATINDEX, MIAR, OCLC WORLDCAT, RESH, REDIB, RILM CORE JOURNALS, SUDOC, ULRICHS, ESTA REVISTA ESTA PUBLICADA CON EL APOYO INSTITUCIONAL DE REDIRIS-CONSEJO SUPERIOR DE INVESTIGACIONES CIENTIFICAS Y ES DE ACCESO LIBRE. CREATIVE COMMONS LICENSE 4.O BY 
Tabla 13. Resultados pregunta 3 “A su juicio, ¿a sus alumnos les gusta cantar”

\begin{tabular}{ccc}
\hline & Frecuencia & Porcentaje \\
\hline Mucho & 204 & $66.4 \%$ \\
Bastante & 89 & $29.0 \%$ \\
Poco & 12 & $3.9 \%$ \\
Nada & 0 & $0.0 \%$ \\
NS/NC & 2 & $0.7 \%$ \\
\hline
\end{tabular}

Fuente: elaboración propia

Las respuestas del profesorado en las 3 provincias manifiestan que les gusta mucho o bastante en unos porcentajes muy similares (Sevilla: 98.5\%; Granada: 94.8\% y Almería: 94.5\%) (Tabla 14).

Tabla 14. Relación Provincias - pregunta 3 "A su juicio, ¿a sus alumnos les gusta cantar"

\begin{tabular}{cccc}
\hline & Almería & Granada & Sevilla \\
\hline \multirow{2}{*}{ Mucho } & 113 & 41 & 50 \\
& $62.1 \%$ & $70.7 \%$ & $74.6 \%$ \\
Bastante & 59 & 14 & 16 \\
& $32.4 \%$ & $24.1 \%$ & $23.9 \%$ \\
Poco & 10 & 2 & 0 \\
& $5.5 \%$ & $3.4 \%$ & $0.0 \%$ \\
\hline
\end{tabular}

Fuente: elaboración propia

La variable nivel educativo en el que imparte sus clases el profesorado (significatividad asintótica, $\left.\chi^{2}=0.000\right)$ también ha arrojado datos relevantes en las respuestas contabilizadas en esta pregunta (Tabla 15). Un $63.7 \%$ del total de maestros que señalan que a sus alumnos le gusta mucho cantar corresponde a maestros de Educación Infantil. A medida que subimos en el nivel educativo el número de docentes que afirman que a sus educandos les gusta cantar mucho o bastante va decreciendo.

Tabla 15. Relación Nivel Educativo - pregunta 3 “A su juicio, ¿a sus alumnos les gusta cantar?”

\begin{tabular}{ccccccc}
\hline & Ed. & $1^{\circ} \mathrm{y} 2^{\circ}$ & $3^{\circ} \mathrm{y} 4^{\circ}$ & $5^{\circ}$ y $6^{\circ}$ & Todos & Total \\
\hline Infantil & 109 & 23 & 15 & 4 & 20 & 171 \\
Mucho & $63.7 \%$ & $13.4 \%$ & $8.8 \%$ & $2.3 \%$ & $11.7 \%$ & $100 \%$ \\
& 27 & 18 & 9 & 8 & 7 & 69 \\
Bastante & $39.1 \%$ & $26.1 \%$ & $13.0 \%$ & $11.6 \%$ & $10.1 \%$ & $100 \%$ \\
& 0 & 3 & 4 & 2 & 0 & 9 \\
Poco & $0.0 \%$ & $33.3 \%$ & $44.4 \%$ & $22.2 \%$ & $0.0 \%$ & $100 \%$ \\
& 0 & 0 & 0 & 0 & 0 & 0 \\
Nada & $0.0 \%$ & $0.0 \%$ & $0.0 \%$ & $0.0 \%$ & $0.0 \%$ & $100 \%$ \\
& 136 & 44 & 28 & 14 & 27 & 249 \\
Total & $54.7 \%$ & $17.6 \%$ & $11.2 \%$ & $5.6 \%$ & $10.8 \%$ & $100 \%$ \\
\hline
\end{tabular}

Fuente: elaboración propia

@JUAN RAFAEL MUÑOZ MUÑOZ. THE CONTENT OF THIS ARTICLE IS THE SOLE RESPONSIBILITY OF THE AUTHORS. THE REVISTA ELECTRÓNICA DE LEEME AND UNIVERSITAT DE VALĖNCIA ARE NOT LIABLE FOR ANY LEGAL ACTIONS THAT MAY ARISE INVOLVING THE ARTICLE'S CONTENT. REVISTA ELECTRÓNICA DE LEEME -LISTA ELECTRÓNICA EUROPEA DE MÚSICA EN LA EDUCACIÓN.HTTP://OJS.UV.ES/INDEX/PHP/LEEME/INDEX. ISSN: 1575-9563. EDITORES: UNIVERSIDAD DE VALENCIA Y JESÚS TEJADA GIMÉNEZ. VISIBILIDAD DE ESTA REVISTA: SCOPUS, EMERGING SOURCES CITATION INDEX (CLARIVATE), EBSCO, CINDOC (CSIC), CITEFACTOR, COPAC, DIALNET, DICE (CSIC), DOAJ, E-REVISTAS (CSIC), EBSCO PREMIER, ERIH+, GALE CENGAGE LEARNING, IN-RECS, IRESIE, LATINDEX, MIAR, OCLC WORLDCAT, RESH, REDIB, RILM CORE JOURNALS, SUDOC, ULRICHS, ESTA REVISTA ESTA PUBLICADA CON EL APOYO INSTITUCIONAL DE REDIRIS-CONSEJO SUPERIOR DE INVESTIGACIONES CIENTIFICAS Y ES DE ACCESO LIBRE. CREATIVE COMMONS LICENSE 4.O BY 
La variable titulación también ha condicionado los resultados obtenidos en esta pregunta (significatividad asintótica $\chi^{2}=0.000$ ). Así, las maestras de Ed. Infantil son los que en su mayoría afirman que a sus alumnos les gusta cantar en clase mucho o bastante. Por el contrario, no se aprecia una mayoría tan importante de maestros de Ed. Primaria que opinen lo mismo.

Tabla 16. Relación Titulación - pregunta 3 "A su juicio, ¿a sus alumnos les gusta cantar”

\begin{tabular}{cccccc}
\hline & Ed. Infantil & Ed. Primaria & Ed. Musical & Otras & Total \\
\hline \multirow{2}{*}{ Mucho } & 104 & 20 & 25 & 37 & 186 \\
& $55.9 \%$ & $10.8 \%$ & $13.4 \%$ & $19.9 \%$ & $100 \%$ \\
Bastante & 32 & 13 & 16 & 20 & 81 \\
& $39.5 \%$ & $16.0 \%$ & $19.8 \%$ & $24.7 \%$ & $100 \%$ \\
Poco & 1 & 2 & 3 & 5 & 11 \\
& $9.1 \%$ & $18.2 \%$ & $27.3 \%$ & $45.4 \%$ & $100 \%$ \\
Nada & 0 & 0 & 0 & 0 & 0 \\
& $0.0 \%$ & $0.0 \%$ & $0.0 \%$ & $100 \%$ & $100 \%$ \\
Total & 137 & 35 & 44 & 62 & 278 \\
& $49.3 \%$ & $12.6 \%$ & $15.8 \%$ & $22.3 \%$ & $100 \%$ \\
\hline
\end{tabular}

Fuente: elaboración propia

Para conocer la importancia que según los maestros la escuela y el entorno dan a la actividad de canto se ha realizado la siguiente pregunta: ¿Qué importancia considera que le dan al hecho de cantar?:

1.- Importancia que le da al hecho de cantar el maestro. De los 307 maestros encuestados más de la mitad de ellos, un 54.4\% señalan que es mucha la importancia que le dan al hecho de cantar y el 33.9\% que es bastante. Sólo 1 maestro no le da ninguna importancia (Tabla 17).

Tabla 17. Resultados pregunta 4, a) La importancia que le da al hecho de cantar el maestro encuestado

\begin{tabular}{ccc}
\hline & Frecuencia & Porcentaje \\
\hline Mucha & 167 & $54.4 \%$ \\
Bastante & 104 & $33.9 \%$ \\
Poca & 29 & $9.4 \%$ \\
Ninguna & 1 & $0.3 \%$ \\
NS/NC & 6 & $2.0 \%$ \\
\hline
\end{tabular}

Fuente: elaboración propia

El análisis de los cuestionarios indica que más del $87.0 \%$ de los maestros de las tres provincias dan mucha o bastante importancia al hecho de cantar (Sevilla: 91.0\%; Granada: 87.9\%; y Almería: 87.3\%). Como puede verse en la Tabla 18:

@JUAN RAFAEL MUÑOZ MUÑOZ. THE CONTENT OF THIS ARTICLE IS THE SOLE RESPONSIBILITY OF THE AUTHORS. THE REVISTA ELECTRÓNICA DE LEEME AND UNIVERSITAT DE VALĖNCIA ARE NOT LIABLE FOR ANY LEGAL ACTIONS THAT MAY ARISE INVOLVING THE ARTICLE'S CONTENT. REVISTA ELECTRÓNICA DE LEEME -LISTA ELECTRÓNICA EUROPEA DE MÚSICA EN LA EDUCACIÓN.HTTP://OJS.UV.ES/INDEX/PHP/LEEME/INDEX. ISSN: 1575-9563. EDITORES: UNIVERSIDAD DE VALENCIA Y JESÚS TEJADA GIMÉNEZ. VISIBILIDAD DE ESTA REVISTA: SCOPUS, EMERGING SOURCES CITATION INDEX (CLARIVATE), EBSCO, CINDOC (CSIC), CITEFACTOR, COPAC, DIALNET, DICE (CSIC), DOAJ, E-REVISTAS (CSIC), EBSCO PREMIER, ERIH+, GALE CENGAGE LEARNING, IN-RECS, IRESIE, LATINDEX, MIAR, OCLC WORLDCAT, RESH, REDIB, RILM CORE JOURNALS, SUDOC, ULRICHS, ESTA REVISTA
PUBLICADA CON EL APOYO INSTITUCIONAL DE REDIRIS-CONSEJO SUPERIOR DE INVESTIGACIONES CIENTIFICAS Y ES DE ACCESO LIBRE. CREATIVE COMMONS LICENSE 4.0 BY 
Tabla 18. Relación Provincias - pregunta 4, a) La importancia que le da al hecho de cantar el maestro encuestado

\begin{tabular}{cccc}
\hline & Almería & Granada & Sevilla \\
\hline Mucha & 98 & 34 & 35 \\
& $53.8 \%$ & $58.6 \%$ & $52.2 \%$ \\
Bastante & 61 & 17 & 26 \\
& $33.5 \%$ & $29.3 \%$ & $38.8 \%$ \\
Poca & 22 & 5 & 2 \\
& $12.1 \%$ & $8.6 \%$ & $3.0 \%$ \\
Ninguna & 0 & 1 & 0 \\
& $0.0 \%$ & $1.7 \%$ & $0.0 \%$ \\
NS/NC & 1 & 1 & 4 \\
& $0.5 \%$ & $1.7 \%$ & $6.0 \%$ \\
Totales & 182 & 58 & 67 \\
& $100 \%$ & $100 \%$ & $100 \%$ \\
\hline
\end{tabular}

Fuente: elaboración propia

2.- Importancia que le dan al hecho de cantar los compañeros. El segundo apartado pretende obtener la información de los encuestados sobre la consideración e importancia que, a su juicio, le otorgan sus compañeros al hecho de cantar. Casi el 50\% de los entrevistados afirman que le dan bastante importancia y un $19.9 \%$ le dan mucha importancia. Curiosamente, el $23.5 \%$ de maestros indican que sus compañeros dan poca importancia al hecho de cantar (Tabla 19).

Tabla 19. Resultados pregunta 4, b) La importancia que le da al hecho de cantar los compañeros

\begin{tabular}{ccc}
\hline & Frecuencia & Porcentaje \\
\hline Mucha & 61 & $19.9 \%$ \\
Bastante & 143 & $46.6 \%$ \\
Poca & 72 & $23.5 \%$ \\
Ninguna & 2 & $0.7 \%$ \\
NS/NC & 29 & $9.4 \%$ \\
\hline
\end{tabular}

Fuente: elaboración propia

Hay que destacar, a la vista de los datos expuestos, que los maestros encuestados consideran que sus compañeros conceden bastante importancia al hecho de cantar (Tabla 20). Sin embargo, no se puede obviar que 72 de los encuestados, consideran que sus compañeros le dan poca importancia. En cuanto a los resultados por provincias, los datos siguen siendo significativos al considerar la suma de las respuestas mucho y bastante (Sevilla: 67.1\%; Almería: 66, 5\% y Granada: 65.5\%).

Tabla 20. Relación Provincias - pregunta 4, b) La importancia que le da al hecho de cantar los compañeros

\begin{tabular}{cccc}
\hline & Almería & Granada & Sevilla \\
\hline \multirow{2}{*}{ Mucha } & 41 & 9 & 11 \\
& $22.5 \%$ & $15.5 \%$ & $16.4 \%$ \\
\hline
\end{tabular}

@JUAN RAFAEL MUÑOZ MUÑOZ. THE CONTENT OF THIS ARTICLE IS THE SOLE RESPONSIBILITY OF THE AUTHORS. THE REVISTA ELECTRÓNICA DE LEEME AND UNIVERSITAT DE VALËNCIA ARE NOT LABLE FOR ANY LEGA ACTIONS THAT MAY ARISE INVOLVING THE ARTICLE'S CONTENT. REVISTA ELECTRONICA DE LEEME - LISTA ELECTRÓNICA EUROPEA DE MÚSICA EN LA EDUCACIÓN-HTTP://OJS.UV.ES/INDEX/PHP/LEEME/INDEX. ISSN: 1575-9563. EDITORES: UNIVERSIDAD DE VALENCIA Y JESÚS TEJADA GIMÉNEZ. VISIBILIDAD DE ESTA REVISTA: SCOPUS, EMERGING SOURCES CITATION INDEX (CLARIVATE), EBSCO, CINDOC (CSIC), CITEFACTOR, COPAC, DIALNET, DICE (CSIC), DOAJ, E-REVISTAS (CSIC), EBSCO PREMIER, ERIH+, GALE CENGAGE LEARNING, IN-RECS, IRESIE, LATINDEX, MIAR, OCLC WORLDCAT, RESH, REDIB, RILM CORE JOURNALS, SUDOC, ULRICHS, ESTA REVISTA ESTÁ PUBLICADA CON EL APOYO INSTITUCIONAL DE REDIRIS-CONSEJO SUPERIOR DE INVESTIGACIONES CIENTIFICAS Y ES DE ACCESO LIBRE. CREATIVE COMMONS LICENSE 4.O BY 


\begin{tabular}{cccc}
\hline Bastante & 80 & 29 & 34 \\
& $44.0 \%$ & $50.0 \%$ & $50.7 \%$ \\
Poca & 45 & 14 & 13 \\
& $24.7 \%$ & $24.1 \%$ & $19.4 \%$ \\
Ninguna & 1 & 0 & 1 \\
& $0.5 \%$ & $0.0 \%$ & $1.5 \%$ \\
NS/NC & 15 & 6 & 8 \\
& $8.2 \%$ & $10.3 \%$ & $11.9 \%$ \\
Totales & 182 & 58 & 67 \\
& $100 \%$ & $100 \%$ & $100 \%$ \\
\hline
\end{tabular}

Fuente: elaboración propia

3.- Importancia que le dan al hecho de cantar los padres. Los datos obtenidos, en esta ocasión, están más repartidos puesto que el $45 \%$ de los maestros señalan que los padres le dan mucha o bastante importancia al hecho de cantar, mientras que más del $47 \%$ de los maestros encuestados opinan que le conceden poca o ninguna importancia (Tabla 21):

Tabla 21. Resultados pregunta 4, c) La importancia que le dan al hecho de cantar los padres

\begin{tabular}{ccc}
\hline & Frecuencia & Porcentaje \\
\hline Mucha & 29 & $9.4 \%$ \\
Bastante & 111 & $36.2 \%$ \\
Poca & 135 & $44.0 \%$ \\
Ninguna & 11 & $3.6 \%$ \\
NS/NC & 21 & $6.8 \%$ \\
\hline
\end{tabular}

Fuente: elaboración propia

Lo más significativo de las respuestas recogidas por provincias (Tabla 22) es poder constatar cómo, a juicio de casi la mitad de los maestros encuestados, los padres conceden poca importancia al hecho de que sus hijas y sus hijos canten.

Tabla 22. Relación Provincias - pregunta 4, c) La importancia que le dan al hecho de cantar los padres

\begin{tabular}{cccc}
\hline & Almería & Granada & Sevilla \\
\hline Mucha & 15 & 6 & 8 \\
& $8.2 \%$ & $10.3 \%$ & $11.9 \%$ \\
Bastante & 66 & 22 & 23 \\
& $36.3 \%$ & $37.9 \%$ & $34.3 \%$ \\
Poca & 83 & 24 & 28 \\
& $45.6 \%$ & $41.4 \%$ & $41.8 \%$ \\
Ninguna & 7 & 2 & 2 \\
& $3.8 \%$ & $3.4 \%$ & $3.0 \%$ \\
NS/NC & 11 & 4 & 6 \\
& $6.0 \%$ & $6.9 \%$ & $9.0 \%$ \\
Totales & 182 & 58 & 67 \\
& $100 \%$ & $100 \%$ & $100 \%$ \\
\hline
\end{tabular}

Fuente: elaboración propia

@JUAN RAFAEL MUÑOZ MUÑOZ. THE CONTENT OF THIS ARTICLE IS THE SOLE RESPONSIBILITY OF THE AUTHORS. THE REVISTA ELECTRÓNICA DE LEEME AND UNIVERSITAT DE VALËNCIA ARE NOT LABLE FOR ANY LEGALACTONS THAT MAY ARISE INYOLVING THE ARTICLE'S CONTENT. REVISTA ELECTRÓNCA DE LEEME - LISTA ELECTRÓNICA EUROPEA DE MÚSICA EN LA EDUCACIÓN-HTTP://OJS.UV.ES/INDEX/PHP/LEEME/INDEX. ISSN: 1575-9563. EDITORES: UNIVERSIDAD DE VALENCIA Y JESÚS TEJADA GIMÉNEZ. VISIBILIDAD DE ESTA REVISTA: SCOPUS, EMERGING SOURCES CITATION INDEX (CLARIVATE), EBSCO, CINDOC (CSIC), CITEFACTOR, COPAC, DIALNET, DICE (CSIC), DOAJ, E-REVISTAS (CSIC), EBSCO PREMIER, ERIH+, GALE CENGAGE LEARNING, IN-RECS, IRESIE, LATINDEX, MIAR, OCLC WORLDCAT, RESH, REDIB, RILM CORE JOURNALS, SUDOC, ULRICHS, ESTA REVISTA ESTÁ PUBLICADA CON EL APOYO INSTITUCIONAL DE REDIRIS-CONSEJO SUPERIOR DE INVESTIGACIONES CIENTIFICAS Y ES DE ACCESO LIBRE. CREATIVE COMMONS LICENSE 4.O BY 
4.- Importancia que las editoriales otorgan al hecho de cantar. El cuarto apartado permitirá conocer la valoración que el profesorado tiene sobre la importancia que dan las editoriales al hecho de cantar (Tabla 23).

Tabla 23. Resultados pregunta 4, d) La importancia que le dan al hecho de cantar las editoriales

\begin{tabular}{ccc}
\hline & Frecuencia & Porcentaje \\
\hline Mucha & 23 & $7.5 \%$ \\
Bastante & 118 & $38.4 \%$ \\
Poca & 128 & $41.7 \%$ \\
Ninguna & 8 & $2.6 \%$ \\
NS/NC & 30 & $9.8 \%$ \\
\hline
\end{tabular}

Fuente: elaboración propia

A juicio de más del $45 \%$ del profesorado, las editoriales dan mucha o bastante importancia a esta actividad (Tabla 24). En cambio, más del $44 \%$ de los entrevistados señala que las editoriales consideran poco o nada importante cantar. Por provincias, el profesorado de la provincia de Sevilla opina que las editoriales dan bastante importancia, concretamente un $46.3 \%$, al hecho de cantar. Sin embargo, el profesorado de las provincias de Almería y Granada considera que las editoriales otorgan poca importancia a cantar.

Tabla 24. Relación Provincias - pregunta 4, d) La importancia que le dan al hecho de cantar las editoriales

\begin{tabular}{cccc}
\hline & Almería & Granada & Sevilla \\
\hline Mucha & 10 & 5 & 8 \\
& $5.5 \%$ & $8.6 \%$ & $11.9 \%$ \\
Bastante & 67 & 20 & 31 \\
& $36.8 \%$ & $34.5 \%$ & $46.3 \%$ \\
Poca & 85 & 27 & 16 \\
& $46.7 \%$ & $46.6 \%$ & $23.9 \%$ \\
Ninguna & 5 & 1 & 2 \\
& $2.7 \%$ & $1.7 \%$ & $3.0 \%$ \\
NS/NC & 15 & 5 & 10 \\
& $8.2 \%$ & $8.6 \%$ & $14.9 \%$ \\
Totales & 182 & 58 & 67 \\
& $100 \%$ & $100 \%$ & $100 \%$ \\
\hline
\end{tabular}

Fuente: elaboración propia

5.- Importancia que la administración educativa da al hecho de cantar. En esta ocasión, como se puede apreciar en la siguiente Tabla 25, hay una mayor coincidencia de opinión entre las maestras y los maestros: más del $67 \%$ del profesorado entrevistado afirma que la administración concede poca o ninguna importancia a esta actividad. Únicamente el $18 \%$ de los maestros señalan que la administración da mucha o bastante importancia al hecho de cantar.

@JUAN RAFAEL MUÑOZ MUÑOZ. THE CONTENT OF THIS ARTICLE IS THE SOLE RESPONSIBILITY OF THE AUTHORS. THE REVISTA ELECTRÓNICA DE LEEME AND UNIVERSITAT DE VALËNCIA ARE NOT LABE FOR ANY LEGA ACTIONS THAT MAY ARISE INNOLVING THE ARTICLE'S CONTENT. REVISTA ELECTRÓNICA DE LEEME - LISTA ELECTRÓNICA EUROPEA DE MÚSICA EN LA EDUCACIÓN-HTTP://OJS.UV.ES/INDEX/PHP/LEEME/INDEX. ISSN: 1575-9563. EDITORES: UNIVERSIDAD DE VALENCIA Y JESÚS TEJADA GIMÉNEZ. VISIBILIDAD DE ESTA REVISTA: SCOPUS, EMERGING SOURCES CITATION INDEX (CLARIVATE), EBSCO, CINDOC (CSIC), CITEFACTOR, COPAC, DIALNET, DICE (CSIC), DOAJ, E-REVISTAS (CSIC), EBSCO PREMIER, ERIH+, GALE CENGAGE LEARNING, IN-RECS, IRESIE, LATINDEX, MIAR, OCLC WORLDCAT, RESH, REDIB, RILM CORE JOURNALS, SUDOC, ULRICHS, ESTA REVISTA ESTÁ PUBLICADA CON EL APOYO INSTITUCIONAL DE REDIRIS-CONSEJO SUPERIOR DE INVESTIGACIONES CIENTIFICAS Y ES DE ACCESO LIBRE. CREATIVE COMMONS LICENSE 4.O BY 
Tabla 25. Resultados pregunta 4, e) La importancia que le da al hecho de cantar la administración educativa

\begin{tabular}{ccc}
\hline & Frecuencia & Porcentaje \\
\hline Mucha & 6 & $2.0 \%$ \\
Bastante & 49 & $16.0 \%$ \\
Poca & 172 & $56.0 \%$ \\
Ninguna & 36 & $11.7 \%$ \\
NS/NC & 44 & $14.3 \%$ \\
\hline
\end{tabular}

Fuente: elaboración propia

El análisis de los datos obtenidos por provincias (Tabla 26) permite comprobar que el profesorado encuestado coincide en afirmar que la administración le da poca importancia al hecho de cantar.

Tabla 26. Relación Provincias - pregunta 4, e) La importancia que le da al hecho de cantar la administración educativa

\begin{tabular}{cccc}
\hline & Almería & Granada & Sevilla \\
\hline Mucha & 2 & 1 & 3 \\
& $1.1 \%$ & $1.7 \%$ & $4.5 \%$ \\
Bastante & 33 & 6 & 10 \\
& $18.1 \%$ & $10.3 \%$ & $14.9 \%$ \\
Poca & 102 & 36 & 34 \\
& $56.0 \%$ & $62.1 \%$ & $50.7 \%$ \\
Ninguna & 20 & 6 & 10 \\
& $11.0 \%$ & $10.3 \%$ & $14.9 \%$ \\
NS/NC & 25 & 9 & 10 \\
& $13.7 \%$ & $15.5 \%$ & $14.9 \%$ \\
Totales & 182 & 58 & 67 \\
& $100 \%$ & $100 \%$ & $100 \%$ \\
\hline
\end{tabular}

Fuente: elaboración propia

6.- Importancia que la sociedad en general concede al hecho de cantar. El sexto, y último, de los apartados de esta pregunta se refiere a la importancia que concede la sociedad, en general, al hecho de cantar (Tabla 27):

Tabla 27. Resultados - pregunta 4, f) La importancia que le da al hecho de cantar la sociedad en general

\begin{tabular}{ccc}
\hline & Frecuencia & Porcentaje \\
\hline Mucha & 16 & $5.2 \%$ \\
Bastante & 60 & $19.5 \%$ \\
Poca & 171 & $55.7 \%$ \\
Ninguna & 18 & $5.9 \%$ \\
NS/NC & 42 & $13.7 \%$ \\
\hline
\end{tabular}

Fuente: elaboración propia

De nuevo, ante esta pregunta, se ha encontrado una opinión unánime en las respuestas del profesorado: más del $60 \%$ de los maestros indican que la sociedad concede poca o ninguna @JUAN RAFAEL MUÑOZ MUÑOZ. THE CONTENT OF THIS ARTICLE IS THE SOLE RESPONSIBILITY OF THE AUTHORS. THE REVISTA ELECTRÓNICA DE LEEME AND UNIVERSITAT DE
VALĖNCIA ARE NOT LIABLE FOR ANY LEGAL ACTIONS THAT MAY ARISE INVOLVING THE ARTICLE'S CONTENT. REVISTA ELECTRÓNICA DE LEEME - LISTA ELECTRÓNICA EUROPEA DE MÚSICA EN LA EDUCACIÓN-HTTP://OJS.UV.ES/INDEX/PHP/LEEME/INDEX. ISSN: 1575-9563. EDITORES: UNIVERSIDAD DE VALENCIA Y JESÚS TEJADA GIMÉNEZ. VISIBILIDAD DE ESTA REVISTA: SCOPUS, EMERGING SOURCES CITATION INDEX (CLARIVATE), EBSCO, CINDOC (CSIC), CITEFACTOR, COPAC, DIALNET, DICE (CSIC), DOAJ, E-REVISTAS (CSIC), EBSCO PREMIER, ERIH+, GALE CENGAGE LEARNING, IN-RECS, IRESIE, LATINDEX, MIAR, OCLC WORLDCAT, RESH, REDIB, RILM CORE JOURNALS, SUDOC, ULRICHS, ESTA REVISTA ESTÁ PUBLICADA CON EL APOYO INSTITUCIONAL DE REDIRIS-CONSEJO SUPERIOR DE INVESTIGACIONES CIENTIFICAS Y ES DE ACCESO LIBRE. CREATIVE COMMONS LICENSE 4.O BY 
importancia a cantar. Y, algo más del $24 \%$ de los entrevistados opina que la sociedad otorga mucha o bastante importancia al canto. En las tres provincias coincide en señalar que la sociedad en general da poca importancia a cantar (Tabla 28):

Tabla 28. Relación Provincias - pregunta 4, f) La importancia que le da al hecho de cantar la sociedad en general

\begin{tabular}{cccc}
\hline & Almería & Granada & Sevilla \\
\hline \multirow{2}{*}{ Mucha } & 8 & 3 & 5 \\
& $4.4 \%$ & $5.2 \%$ & $7.5 \%$ \\
Bastante & 37 & 9 & 14 \\
& $20.3 \%$ & $15.5 \%$ & $20.9 \%$ \\
Poca & 99 & 36 & 36 \\
& $54.4 \%$ & $62.1 \%$ & $53.7 \%$ \\
Ninguna & 13 & 3 & 2 \\
& $7.1 \%$ & $5.2 \%$ & $3.0 \%$ \\
NS/NC & 25 & 7 & 10 \\
& $13.7 \%$ & $12.1 \%$ & $14.9 \%$ \\
Totales & 182 & 58 & 67 \\
& $100 \%$ & $100 \%$ & $100 \%$ \\
\hline
\end{tabular}

Fuente: elaboración propia

\section{Discusión y conclusiones}

El análisis y la valoración de los resultados obtenidos ofrece la visión del profesorado encuestado sobre la presencia del canto en sus aulas y la importancia que le otorga al hecho de cantar. Respecto al primero de los objetivos específicos establecidos, se puede comprobar que a una amplia mayoría del profesorado le gusta cantar en su aula. Paralelamente, el profesorado encuestado opina que a su alumnado también les gusta cantar. Lo más significativo de estos resultados es que en ambos casos las respuestas a favor del canto han sido superiores al $90 \%$. Esta disposición hacia el canto es mayor en los cursos de Ed. Infantil, y lo sigue siendo en primero, segundo, tercero y cuarto de Ed. Primaria, pero se reduce en quinto y sexto. Estos resultados vienen a apoyar la idea de que el infante muestra una disposición positiva para cantar (Agosti y Rapp, 1988).

En cuanto al segundo de los objetivos específicos de este estudio, referido a la periodicidad del canto en el aula, a juicio del profesorado, la presencia de esta actividad en las clases es diaria o al menos habitual. Si atendemos al nivel educativo, se puede constatar que esta presencia es mayor en Educación Infantil y es menor en los cursos quinto y sexto (Cámara, 2005). Como ocurre con los resultados del objetivo anterior, a medida que se va ascendiendo de curso la presencia del canto en clase va disminuyendo. Esto podría tener una relación directa con la idea que ha sido desarrollada por diferentes autores que consideran que a partir de los diez años es más difícil implicar al alumnado en la actividad de canto (Vander Ark, Nolin y Newman, 1980; Bowles, 1998). Considerando la especialidad del profesorado, las maestras de

@JUAN RAFAEL MUÑOZ MUÑOZ. THE CONTENT OF THIS ARTICLE IS THE SOLE RESPONSIBILITY OF THE AUTHORS. THE REVISTA ELECTRÓNICA DE LEEME AND UNIVERSITAT DE VALĖNCIA ARE NOT LIABLE FOR ANY LEGAL ACTIONS THAT MAY ARISE INVOLVING THE ARTICLE'S CONTENT. REVISTA ELECTRÓNICA DE LEEME -LISTA ELECTRÓNICA EUROPEA DE MÚSICA EN LA EDUCACIÓN.HTTP://OJS.UV.ES/INDEX/PHP/LEEME/INDEX. ISSN: 1575-9563. EDITORES: UNIVERSIDAD DE VALENCIA Y JESÚS TEJADA GIMÉNEZ. VISIBILIDAD DE ESTA REVISTA: SCOPUS, EMERGING SOURCES CITATION INDEX (CLARIVATE), EBSCO, CINDOC (CSIC), CITEFACTOR, COPAC, DIALNET, DICE (CSIC), DOAJ, E-REVISTAS (CSIC), EBSCO PUBLICADA CON EL APOYO INSTITUCIONAL DE REDIRIS-CONSEJO SUPERIOR DE INVESTIGACIONES CIENTIFICAS Y ES DE ACCESO LIBRE. CREATIVE COMMONS LICENSE 4.O BY 
Educación Infantil son las que más cantan en el aula, seguidas de las maestras y los maestros especialistas en Educación Musical y de las maestras y los maestros de Educación Primaria.

El tercer objetivo específico planteado pretendía comprobar la importancia que se le concede al canto en el aula. Los datos obtenidos señalan que más del $88 \%$ del profesorado afirma que es mucha o al menos bastante importante la actividad de canto en su aula. De igual modo, señalan que para más de un $66 \%$ de sus compañeros, la importancia que conceden a esta actividad también es mucha o bastante. Por otra parte, el $44 \%$ profesorado encuestado opina que los padres le conceden poca importancia al hecho de cantar en clase. Estos resultados vienen a reafirmar el dato de que las madres y los padres cada vez cantan menos a sus hijas e hijos (Poch, 2005). Analizando la importancia que conceden las editoriales al canto se puede observar como la opinión está dividida entre los encuestados. El 45,9\% de los maestros piensan que las editoriales le conceden mucha o bastante importancia al canto, mientras que para el 41,7\%, la importancia es poca. Es posible que esas opiniones tengan que ver con la diversidad de oferta de canciones que presentan las editoriales en los libros de texto de música (Blanco, 2011). Para más del $50 \%$ del profesorado encuestado, la administración educativa otorga poca importancia al hecho de cantar. Aproximadamente, ese mismo porcentaje de maestros opinan que la importancia que le concede la sociedad en general al canto en el aula también es poca.

Teniendo en cuenta todo lo expuesto respecto a los objetivos específicos y considerando el principal objetivo de ésta investigación, los datos aportados permiten afirmar que el profesorado encuestado valora como muy importante o bastante importante el hecho de cantar en el aula y entienden que sus compañeros tienen esa misma opinión, pero está importancia es menor para los padres. En el ámbito institucional, la importancia del canto sigue descendiendo para las editoriales, y es aún más baja, o menos importante, para la administración educativa y la sociedad en general.

Estos resultados hacen necesario que se insista en destacar la importancia del canto y sus beneficios en la formación integral del infante. Para ello, sería conveniente desarrollar algunas iniciativas que podrían ayudar en esa labor. Entre ellas, se puede potenciar la utilización de las canciones como ejes de globalización en el desarrollo de proyectos educativos en el aula para poner de relieve su importancia (Pascual, 2006). La participación de las familias en actividades de recopilación de canciones populares infantiles puede utilizarse como medio para incentivar que tanto los abuelos como los padres se animen a cantar en casa. Con ello se estaría favoreciendo el cuidado y mantenimiento de muchas canciones populares infantiles que forman parte de la tradición oral de nuestro país (Cerrillo, 2005; Costa, 2003).

Por otra parte, la selección por parte del profesorado de aquellos textos editoriales que valoren la canción, incluyendo una amplia variedad de canciones en cada uno de los cursos, puede llamar la atención de las editoriales para cuidar la presencia de las canciones en sus textos. De cara a la administración educativa y a la sociedad en general, las iniciativas pueden

@JUAN RAFAEL MUÑOZ MUÑOZ. THE CONTENT OF THIS ARTICLE IS THE SOLE RESPONSIBILITY OF THE AUTHORS. THE REVISTA ELECTRÓNICA DE LEEME AND UNIVERSITAT DE VALĖNCIA ARE NOT LIABLE FOR ANY LEGAL ACTIONS THAT MAY ARISE INVOLVING THE ARTICLE'S CONTENT. REVISTA ELECTRÓNICA DE LEEME -LISTA ELECTRÓNICA EUROPEA DE MÚSICA EN LA EDUCACIÓN-HTTP://OJS.UV.ES/INDEX/PHP/LEEME/INDEX. ISSN: 1575-9563. EDITORES: UNIVERSIDAD DE VALENCIA Y JESÚS TEJADA GIMÉNEZ. VISIBILIDAD DE ESTA REVISTA: SCOPUS, EMERGING SOURCES CITATION INDEX (CLARIVATE), EBSCO, CINDOC (CSIC), CITEFACTOR, COPAC, DIALNET, DICE (CSIC), DOAJ, E-REVISTAS (CSIC), EBSCO PUBLICADA CON EL APOYO INSTITUCIONAL DE REDIRIS-CONSEJO SUPERIOR DE INVESTIGACIONES CIENTIFICAS Y ES DE ACCESO LIBRE. CREATIVE COMMONS LICENSE 4.O BY 
encaminarse a hacer visible esa importancia con la realización de actividades de los centros las acciones de canto en el marco de las fiestas, celebraciones y conmemoraciones que se trabajan a lo largo de cada curso (Garrido, 2011). Del mismo modo, se debe potenciar la utilización de las canciones en tareas de clase que pretendan fomentar la inclusión en las aulas (González Martín y Muñoz Muñoz, 2019). La disponibilidad de un repertorio más amplio de canciones también facilitará el acercamiento del alumnado al estudio de contenidos de otras áreas de conocimiento (Gul y Bozcaya, 2015). Además, este repertorio debe contemplar los tipos de música que el infante prefiere (Arriaga, Riaño, Cabedo y Berbel, 2017). Se considera que con todas estas iniciativas se ayudaría a mejorar la importancia que deben otorgar al canto las compañeras y los compañeros, las madres y los padres, las editoriales, la administración educativa y la sociedad en general. De mismo modo, también ayudarían a que todas ellas y todos ellos tomaran conciencia de las posibilidades que ofrece el canto en la escuela como medio para el desarrollo de capacidades que no son exclusivamente musicales y como medio para el desarrollo personal y social, el tratamiento de las emociones y la educación en valores.

Con todo ello, se pretende llamar la atención de todas las personas e instituciones que intervienen en la educación del infante en el aula para que se le dé al canto la consideración y la importancia que merece en el ámbito de la formación musical, en particular, y de la formación integral, en general. Pensando en futuras líneas de investigación que se pueden plantear, a partir de los datos aportados pueden sugerirse, entre otras, las siguientes: análisis de los repertorios de canciones en las aulas, propuestas de intervenciones didácticas a partir de canciones vinculadas con cualquiera de las áreas de conocimiento, o diseño de actividades con canciones favorecedoras de la educación en valores y la inclusión en el aula. Con todas ellas, se estaría contribuyendo a la toma de conciencia de la importancia que tienen las canciones en las aulas y fomentando su utilización con amplias y diversas finalidades educativas.

\section{Referencias}

Agosti, C. y Rapp, C.H. (1988). El niño, el mundo sonoro y la música. Alcoy: Marfil.

Arriaga, C., Riaño, M.E., Cabedo, A. y Berbel, N. (2017). Songs are taught, songs are learnt: musical preferences in early childhood. Music Education Research, 19, 309-326. doi: $10.1080 / 14613808.2016 .1214694$

Bernal, J. y Calvo, M.L. (2004). Didáctica de la Música: la voz y sus recursos. Repertorio de canciones y melodías para la escuela. Málaga: Aljibe.

Bernabé, M. (2013). La intervención educativa musical en contextos pluriculturales. Hekademos, 14, 29-39.

Bisquerra, R. (2011). Educación emocional: propuestas para educadores y familias. Bilbao: 
Desclée de Brouwer.

Blanco. E (2011). La canción infantil en la educación infantil y primaria. Las tic como recurso didáctico en la clase de música. Salamanca: Ediciones Universidad de Salamanca.

Blaxter, L., Hughes, C. y Tight, M. (2000). Cómo se hace una investigación. Barcelona: Gedisa.

Bowles, Ch. L. (1998). Music Activity Preferences of Elementary Students. Journal of Research in Music Education, 46(2), 193-207.

Cabedo, A. y Arriaga, C. (2016). ¿Música para aprender, música para integrar? Arte y educación en valores en el currículo escolar. Dedica. Revista de Educaçao e Humanidades, 9, 145-160.

Cámara Izagirre, A. (2004). La actividad de cantar en la escuela: una práctica en desuso. Revista de Psicodidáctica, 17, 75-84.

Cámara Izagirre, A. (2005). Actitudes de las niñas y de los niños hacia el canto. Revista Musiker, 14, 101-119.

Casals, A., Carrillo, C. y González-Martín, C. (2014). La música también cuenta: combinando matemáticas y música en el aula. Revista electrónica LEEME. 34, 1-17. Recuperado de https://ojs.uv.es/index.php/LEEME/article/viewFile/9861/9280

Cerrillo, P.C. (2005). La voz de la memoria. Cuenca: Universidad de Castilla-La Mancha.

Cerrillo, P. (2010). ¿Dónde está el niño que yo fui? Madrid: Akal.

Chao, R. (2005). La enseñanza del folklore gallego en los colegios de educación primaria de Galicia. Tesis doctoral inédita. Universidad A Coruña.

Consejería de Educación de la Junta de Andalucía (2016). Recursos Humanos de la red de centros. Profesorado. Personal de adm. y Servicios. Disponible en http://www.juntadeandalucia.es/educacion/webportal/web/estadisticas/recursoshumanos-del-sistema-educativo-en-andalucia

Costa, L. (2003). Práctica pedagógica y música tradicional. Revista electrónica de LEEME, 12, 1-7. Recuperado de https://ojs.uv.es/index.php/LEEME/article/view/9744

Cunha, R. y Lorenzino, L. (2012). The secondary aspects of collective music-making. Research Studies in Music Education, 34(1), 73-88. doi:10.1177/1321103X12439134

Díaz, J. (2007). Música y letra. Valladolid: Ámbito Ediciones.

Díaz, M. (2011). Enfoques, concepciones y metodologías sobre el aprendizaje de la música en la

@JUAN RAFAEL MUÑOZ MUÑOZ. THE CONTENT OF THIS ARTICLE IS THE SOLE RESPONSIBILITY OF THE AUTHORS. THE REVISTA ELECTRÓNICA DE LEEME AND UNIVERSITAT DE VALĖNCIA ARE NOT LIABLE FOR ANY LEGAL ACTIONS THAT MAY ARISE INVOLVING THE ARTICLE'S CONTENT. REVISTA ELECTRÓNICA DE LEEME -LISTA ELECTRÓNICA EUROPEA DE MÚSICA EN LA EDUCACIÓN-HTTP://OJS.UV.ES/INDEX/PHP/LEEME/INDEX. ISSN: 1575-9563. EDITORES: UNIVERSIDAD DE VALENCIA Y JESÚS TEJADA GIMÉNEZ. VISIBILIDAD DE ESTA REVISTA: SCOPUS, EMERGING SOURCES CITATION INDEX (CLARIVATE), EBSCO, CINDOC (CSIC), CITEFACTOR, COPAC, DIALNET, DICE (CSIC), DOAJ, E-REVISTAS (CSIC), EBSCO PUBLICADA CON EL APOYO INSTITUCIONAL DE REDIRIS-CONSEJO SUPERIOR DE INVESTIGACIONES CIENTIFICAS Y ES DE ACCESO LIBRE. CREATIVE COMMONS LICENSE 4.O BY 
etapa de infantil. En M.E. Riaño y M. Díaz (Coords.). Fundamentos musicales y didácticos en la Educación Infantil (pp.55-76). Santander: Ediciones Universidad de Cantabria.

Fautley,M. y Daubney, A. (2018). Inclusion, music education, and what it might mean. British Journal of Music Education, 35(3), 219-221. doi: 10.1017/S0265051701000237

Fox, D. J. (1981). El proceso de investigación en educación. Pamplona: Ediciones de la Universidad de Navarra.

García Ferrando, M. (1985). Socioestadística: introducción a la estadística en sociología. Madrid: Alianza Editorial.

Garrido, D.C. (2011). La educación musical en la educación primaria a partir de las fiestas, celebraciones y conmemoraciones. Eufonía. Didáctica de la Música, 51, 80-90.

Giráldez, A. (2014). Enseñar y aprender en el aula de música. En A. Girádez (Coord.). Didáctica de la Música en Primaria (pp.39-76). Madrid: Síntesis.

González Martín, J. y Muñoz Muñoz, J.R. (2019). Songs as a means of cross-cultural education. The European Proceedings of Social \& Behavioural Sciences, 31, 243-249. doi: 10.15405/epsbs.2019.04.02.31

Gul, G. y Bozkaya, I. (2015). The Efficiency of The Song Repertoire on The Musical Development Level of Pre-School Children Aged Six Years Old. Procedia - Social and Behavioral Sciences, 197, 506-513. doi: 10.1016/j.sbspro.2015.07.177.

Hernández, M.D. (2012). Repertorio vocal de los niños de 0 a 6 años. Eufonía. Didáctica de la Música, 55, 63-71.

López de la Calle, M.A. (2007). La música en centros de educación infantil 3-6 años de Galicia e Inglaterra: un estudio de su presencia y de las prácticas educativas. Tesis doctoral: Universidad Santiago de Compostela. Recuperado de: https://minerva.usc.es/xmlui/handle/10347/2355

Martín Escobar, M.J. (2010). Las canciones infantiles de Transmisión Oral en Murcia durante el siglo XX. Murcia: Universidad de Murcia.

Mcmillan, J.H. y Schumacher, S. (2010). Investigación Educativa. Madrid: Pearson Educación.

Ministerio de Educación, Cultura y Deporte (2016). Estadísticas de la Educación. Recuperado de: http://www.mecd.gob.es/servicios-al-ciudadano-mecd

@JUAN RAFAEL MUÑOZ MUÑOZ. THE CONTENT OF THIS ARTICLE IS THE SOLE RESPONSIBILITY OF THE AUTHORS. THE REVISTA ELECTRÓNICA DE LEEME AND UNIVERSITAT DE VALĖNCIA ARE NOT LIABLE FOR ANY LEGAL ACTIONS THAT MAY ARISE INVOLVING THE ARTICLE'S CONTENT. REVISTA ELECTRÓNICA DE LEEME - LISTA ELECTRÓNICA EUROPEA DE MÚSICA EN LA EDUCACIÓN-HTTP://OJS.UV.ES/INDEX/PHP/LEEME/INDEX. ISSN: 1575-9563. EDITORES: UNIVERSIDAD DE VALENCIA Y JESÚS TEJADA GIMÉNEZ. VISIBILIDAD DE ESTA REVISTA: SCOPUS, EMERGING SOURCES CITATION INDEX (CLARIVATE), EBSCO, CINDOC (CSIC), CITEFACTOR, COPAC, DIALNET, DICE (CSIC), DOAJ, E-REVISTAS (CSIC), EBSCO PREMIER, ERIH+, GALE CENGAGE LEARNING, IN-RECS, IRESIE, LATINDEX, MIAR, OCLC WORLDCAT, RESH, REDIB, RILM CORE JOURNALS, SUDOC, ULRICHS, ESTA REVISTA ESTA PUBLICADA CON EL APOYO INSTITUCIONAL DE REDIRIS-CONSEJO SUPERIOR DE INVESTIGACIONES CIENTIFICAS Y ES DE ACCESO LIBRE. CREATIVE COMMONS LICENSE 4.O BY 
Monks, S. (2003). Adolescent singers and perceptions of vocal identity. British Journal Of Music Education, 20 (3), 243-256. doi: 10.1017/S0265051703005424

Morant, R. (1999). La música popular según el ciclo escolar anual. Una experiencia desarrollada en los centros de formación, innovación y recursos educativos de las comarcas centrales de la Comunidad Valenciana. Eufonía. Didáctica de la Música, 16, 106-109.

Moya, M.V; Hernández, J.R.; Hernández, J.A. y Cozar, R. (2014). La educación musical competencial en España: ¿Necesidad o deseo? Revista Electrónica Educare, 18(3), 237249. doi: $10.15359 /$ ree.18-3.14

Muñoz Muñoz, J. R. (2017). Las canciones basadas en cuentos en el aula de infantil. En D. Madrid y M. Barcia (Ed.). Temas clave de Educación Infantil (0-6 años) (pp.337-356). Madrid: La Muralla.

Oriol, N. (2002). La enseñanza del folclore en los colegios de la Comunidad de Madrid. Eufonía. Didáctica de la Música, 25, 67-88.

Pascual, P. (2006). Didáctica de la Música. Madrid: Pearson.

Phillips, K.H. (1992). Research on the teaching of singing. En R. Colwell (Ed.), Handbook of Research on Music Teaching and Learning (pp.568-576). New York: Schirmer Books.

Poch, D. (2005). Música para disfrutar, música para compartir. La educación musical en la temprana infancia con mamá o papá. Eufonía. Didáctica de la Música, 33, 38-46.

Selfa, E. (2019). "Cantem junts". El impacto de la experiencia musical artística intergeneracional como vehículo canalizador de emociones, valores, motivaciones y aprendizajes. (Tesis doctoral no publicada). Universidad de Valencia, Valencia.

Sigcha, E.M., Constante, M.F., Defaz, Y.P. Trávez, J. y Ceíro, W. (2016). La expresión musical como herramienta para el desarrollo integral infantil. Didasc@lia: Didáctica y Educación, 6, 353-370.

Subirats, M.A. (2007). Zoltán Kodály. En M. Díaz y A. Giráldez (Coords.). Aportaciones teóricas y metodológicas a la educación musical (pp.63-70). Barcelona: Graó.

Tiburcio, E. (2010). Canciones y discusión de dilemas en el desarrollo de valores. Una experiencia de intervención en Escuelas Primarias de la República Dominicana. Revista electrónica de LEEME, 25, 123-148. Recuperado de https://ojs.uv.es/index.php/LEEME/article/view/9809

Vander Ark, S.S., Nolin, W.H. y Newman, I. (1980). Relationships between musical attitudes, self-steem, social status, and grade level of elementary children. Bulletin of the Council

@JUAN RAFAEL MUÑOZ MUÑOZ. THE CONTENT OF THIS ARTICLE IS THE SOLE RESPONSIBILITY OF THE AUTHORS. THE REVISTA ELECTRÓNICA DE LEEME AND UNIVERSITAT DE VALĖNCIA ARE NOT LIABLE FOR ANY LEGAL ACTIONS THAT MAY ARISE INVOLVING THE ARTICLE'S CONTENT. REVISTA ELECTRÓNICA DE LEEME - LSTA ELECTRÓNICA EUROPEA DE MÚSICA EN LA EDUCACIÓN.HTTP://OJS.UV.ES/INDEX/PHP/LEEME/INDEX. ISSN: 1575-9563. EDITORES: UNIVERSIDAD DE VALENCIA Y JESÚS TEJADA GIMÉNEZ. VISIBILIDAD DE ESTA REVISTA: SCOPUS, EMERGING SOURCES CITATION INDEX (CLARIVATE), EBSCO, CINDOC (CSIC), CITEFACTOR, COPAC, DIALNET, DICE (CSIC), DOAJ, E-REVISTAS (CSIC), EBSCO PREMIER, ERIH+, GALE CENGAGE LEARNING, IN-RECS, IRESIE, LATINDEX, MIAR, OCLC WORLDCAT, RESH, REDIB, RILM CORE JOURNALS, SUDOC, ULRICHS, ESTA REVISTA ESTÁ PUBLICADA CON EL APOYO INSTITUCIONAL DE REDIRIS-CONSEJO SUPERIOR DE INVESTIGACIONES CIENTÍFICAS Y ES DE ACCESO LIBRE. CREATIVE COMMONS LICENSE 4.O BY 
revista electrónica

$\mathrm{C} \rightleftharpoons \mathrm{C}$

Electronic Journal of Music in Education

Revista arbitrada de investigación y aplicaciones en Educación Musical
peer revieved journal of research and applications in Music Edícation

ISSN: $1575-9563$ SECCIÓN ARTICULOS: JUAN RAFAEL MUÑOZ MUÑOZ
¿A QUIÉN LE IMPORTA EL CANTTO EN EL AULA? ESTUDIO BASADO EN UN CUESTIONARIO (REVISTA ELECTRÓNICA DE LEEME) NÚMERO 44, PP. 1-23 HTTPS://OJS.UV.ES/INDEX.PHP/LEEME/INDEX
DOI: $10.7203 /$ LEEME.44.15631

for Research in Music Education, 62, 31-41.

Willems, E. (1981). El Valor Humano de la Educación Musical. Barcelona: Paidós.

Zamora, A. (2002). Cantos para jugar y bailar. En J. Beltrán, J. Díaz, A. Pelegrin y A. Zamora. (Eds.), Folklore musical infantil (pp.145-167). Madrid: Akal.

@JUAN RAFAEL MUÑOZ MUÑOZ. THE CONTENT OF THIS ARTICLE IS THE SOLE RESPONSIBILITY OF THE AUTHORS. THE REVISTA ELECTRÓNICA DE LEEME AND UNIVERSITAT DE VALĖNCIA ARE NOT LIABLE FOR ANY LEGAL ACTIONS THAT MAY ARISE INVOLVING THE ARTICLE'S CONTENT. REVISTA ELECTRÓNICA DE LEEME -LISTA ELECTRÓNICA EUROPEA DE MÚSICA EN LA EDUCACIÓN.HTTP://OJS.UV.ES/INDEX/PHP/LEEME/INDEX. ISSN: 1575-9563. EDITORES: UNIVERSIDAD DE VALENCIA Y JESÚS TEJADA GIMÉNEZ. VISIBILIDAD DE ESTA REVISTA: SCOPUS, EMERGING SOURCES CITATION INDEX (CLARIVATE), EBSCO, CINDOC (CSIC), CITEFACTOR, COPAC, DIALNET, DICE (CSIC), DOAJ, E-REVISTAS (CSIC), EBSCO
PREMIER, ERIH+, GALE CENGAGE LEARNING, IN-RECS, IRESIE, LATINDEX, MIAR, OCLC WORLDCAT, RESH, REDIB, RILM CORE JOURNALS, SUDOC, ULRICHS, ESTA REVISTA ESTÁ PUBLICADA CON EL APOYO INSTITUCIONAL DE REDIRIS-CONSEJO SUPERIOR DE INVESTIGACIONES CIENTIFICAS Y ES DE ACCESO LIBRE. CREATIVE COMMONS LICENSE 4.O BY 